\title{
The Effect of Pulse Vaccination and Treatment on SIR Epidemic Model with Media Impact
}

\author{
Zhi-Long He and Lin-Fei Nie \\ College of Mathematics and Systems Science, Xinjiang University, Urumqi 830046, China \\ Correspondence should be addressed to Lin-Fei Nie; lfnie@163.com
}

Received 15 April 2015; Revised 3 June 2015; Accepted 4 June 2015

Academic Editor: Gian I. Bischi

Copyright (C) 2015 Z.-L. He and L.-F. Nie. This is an open access article distributed under the Creative Commons Attribution License, which permits unrestricted use, distribution, and reproduction in any medium, provided the original work is properly cited.

We propose a novel SIR epidemic dynamical control model with media impact, where the state dependent pulse vaccination and medication treatment control strategies are being introduced to prevent the spread of disease at different control threshold values. By using the geometry theory of differential equation and method of successor function, the existence of positive order-1 periodic solution is studied. Further, some sufficient conditions of the orbitally asymptotical stability for positive order-1 periodic solution are given by the analog Poincaré criterion. Furthermore, numerical simulations are carried to illustrate the feasibility of our main results presented here.

\section{Introduction}

Millions of human beings suffer from or die of various infectious diseases every year. For example, malaria, dengue, AIDS, SARS, cholera, Ebola, and avian influenza have a tremendous influence on human health at the last few years. Therefore, controlling infectious diseases has been an increasingly complex issue worldwide. It is well known that vaccination is widely regarded as the most effective measure in preventing such viral infections as rabies, yellow fever, poliovirus, hepatitis $\mathrm{B}$, parotitis, and encephalitis $\mathrm{B}$. The vaccination strategies lead to infectious diseases eradication if the proportion of the successfully vaccinated individuals is larger than a certain critical value, for example, which is approximately equal to $95 \%$ for measles [1]. However, in practice, it is both difficult and expensive to implement vaccination for such a large population coverage.

Recently, pulse vaccination has gained prominent achievement as a result of its highly successful application in the control of poliomyelitis and measles throughout Central and South America. In viewing of this, epidemiological models with pulse vaccination control strategies have been set up and investigated in many literatures (see, e.g., [2-7] and the references therein). Particularly, a theoretical result in this context was obtained by Shulgin et al. [8]. They showed that the infection-free solution can exist and be stable, which implies the disease could be eradicated. d'Onofrio [5] proposed a SEIR epidemic model with pulse vaccination strategy and discussed the local and global asymptotic stabilities of the periodic eradication solution. Röst and Vizi [9] investigated a SIVS model with pulse vaccination strategy, and their main result is that nontrivial endemic periodic solutions are bifurcating from the disease-free periodic solution as a parameter is passing through the threshold value one.

In a real world application, however, the eradication of a disease is sometimes difficult both practically and economically in a short time. So, it is necessary to keep the density of infections at a low level to avoid the spread of the disease. Motivated by this idea, the state dependent pulse control strategy is applied widely to the control of spread of infectious disease due to its economic high efficiency and feasibility nature. For example, a simple SIR model with state dependent pulse control strategies was first considered by Tang et al. [10], and theoretical results showed that the combination of pulse vaccination and treatment (or isolation) is optimal in terms of cost under certain conditions, which depends on the RL (where RL is defined as the number of infected patients such that control actions must be taken in order to avoid economic and social damage), and the existence and stability of periodic solution with the maximum value of the infective being no 
larger than RL are obtained. This implies that disease can be successfully controlled in a local area. Further, Nie et al. $[11,12]$ proposed SIR and SIRS models with state dependent pulse vaccination and analyzed the existence and stability of positive periodic solution using the Poincaré map and the method of qualitative analysis. Additionally, the state dependent pulse control strategy also can be found in many other areas like agricultural production and fishery industry, where the control measures (such as catching, poisoning, releasing the natural enemy, and harvesting) are taken only when the number of populations reaches a threshold value. We refer some of them to [13-16] and the references therein.

On the other hand, we note that people's response to the threat of disease is often relied on the public and private information disseminated widely by the media, such as broadcast reports and network information. Massive news coverage and fast information flow can generate a profound psychological impact on the public. A lot of press coverage and fast information flow about the risk of disease can affect the psychological quality of the masses and further affect people's daily behavior. Therefore, media communications have played an important role in affecting the outcome of infectious disease outbreaks (see, e.g., [17-21] and the references therein).

In this paper, according to the different minds and behaviors of people at the different threat levels and different stages of disease, we propose a novel SIR epidemic model with media coverage by combination of state dependent pulse vaccination for the susceptibles and treatment of the infected at different control threshold values. This paper is structured as follows. In Section 2, a SIR epidemic model with media coverage and state dependent pulse control strategies is constructed, and some basic definitions, preliminaries, and lemmas are given. In Section 3, the existence and stability of positive periodic solution of this model are examined. In Section 4, some numerical simulations are given to illustrate our results. Some concluding remarks are presented in the last section.

\section{Model Formulation and Preliminaries}

Wang and Xiao [20] proposed the following SIR epidemic model with media impact:

$$
\begin{aligned}
\frac{\mathrm{d} S(t)}{\mathrm{d} t} & =\mu-\beta \exp (-\alpha \epsilon I) S I-\mu S, \\
\frac{\mathrm{d} I(t)}{\mathrm{d} t} & =\beta \exp (-\alpha \epsilon I) S I-(\mu+\gamma) I, \\
\frac{\mathrm{d} R(t)}{\mathrm{d} t} & =\gamma I-\mu R
\end{aligned}
$$

with

$$
\epsilon= \begin{cases}0, & \sigma(S, I)<0, \\ 1, & \sigma(S, I)>0,\end{cases}
$$

where $\sigma(S, I)=\sigma(I)=I-I_{c} . S, I$, and $R$ represent the densities or quantities of susceptible, infected, and recovered populations, respectively. All model parameters are

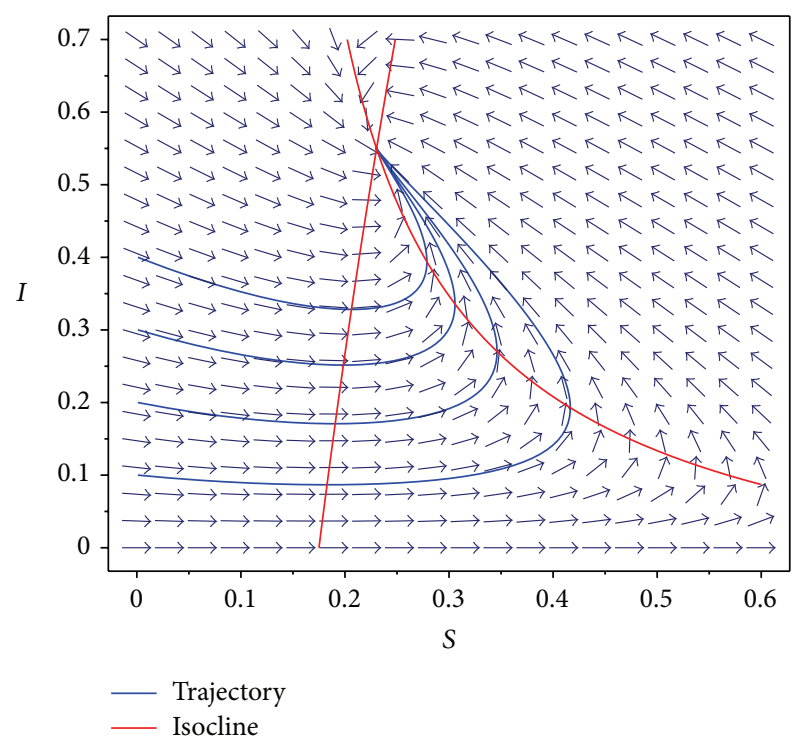

FIgUre 1: The phase space of model (1) with $\epsilon=1$.

positive constants, where $\mu$ is the natural birth/death rate, $\beta$ denotes the basic transmission rate, and $\gamma$ represents the removed/recovered rate. When $I$ increases and reaches a certain level $I_{c}$, mass media start to report information about the disease, including ways of transmission and number of infected individuals, and then the public tries their best to avoid being infected. This consequently lowers the effective contact, resulting in a reduction in transmission rate which is usually represented by $\beta \exp (-\alpha I), 0<\alpha<1$ to reflect the impact of media coverage to the effective contact rate.

From Proposition 2 in [20], authors showed that, for model (1) with $\epsilon=1$, the disease-free equilibrium $E_{0}(1,0,0)$ is globally asymptotically stable if $R_{0}=\beta /(\mu+\gamma)<1$, and the unique endemic equilibrium $E^{*}\left(S^{*}, I^{*}, R^{*}\right)$ is globally asymptotically stable if $R_{0}>1$, where

$$
\begin{aligned}
S^{*} & =\frac{\mu+\gamma}{\beta} \exp \left(\alpha I^{*}\right), \\
I^{*} & =\frac{\mu}{\mu+\gamma}-\frac{1}{\alpha} \operatorname{Lambert} W\left(\frac{\alpha \mu}{\beta} \exp \left(\frac{\alpha \mu}{\mu+\gamma}\right)\right), \\
R^{*} & =1-S^{*}-I^{*}
\end{aligned}
$$

where the Lambert $W$ function is defined to be a multivalued inverse of the function $z \mapsto z e^{z}$ satisfying $W(z) \exp (W(z))=$ $z$.

We assume, throughout this paper, that $\epsilon=1$ and $R_{0}>1$. That is to say, model (1) with $\epsilon=1$ has a unique endemic equilibrium $E^{*}\left(S^{*}, I^{*}, R^{*}\right)$, which is globally asymptotically stable (see Figure 1). To keep the infected density at a low level, we propose a state dependent pulse vaccination for the susceptible patients and treatment for the infected at different control threshold values. Comparing to the disease cycles, the medication for some infectious diseases is relatively short; we suppose that the procedure of medication takes pulse effect when the number of group $I$ reaches the higher threshold value. 
That is, when the density of the infected individuals reaches the higher hazardous threshold value $H_{h}\left(H_{h} \in\left(0, I^{*}\right)\right)$ at time $t_{i}\left(H_{h}\right)$ at the $i$ th time, the vaccination and intense treatment are taken, and the densities of susceptible, infected, and recovered individuals turn very suddenly to a great degree to $(1-p) S\left(t_{i}^{+}\left(H_{h}\right)\right),\left(1-q_{1}\right) I\left(t_{i}^{+}\left(H_{h}\right)\right)$, and $R\left(t_{i}^{+}\left(H_{h}\right)\right)+$ $p S\left(t_{i}^{+}\left(H_{h}\right)\right)+q_{1} I\left(t_{i}^{+}\left(H_{h}\right)\right)$, respectively, where $p \in(0,1)$ and $q_{1} \in(0,1)$ are the vaccination intensity and medication intensification effort, respectively. However, when the density of the susceptible reaches the relatively small threshold value $H_{l}\left(H_{l} \in\left(0,\left(1-q_{1}\right) H_{h}\right)\right)$ at time $t_{j}\left(H_{l}\right)$ at the $j$ th time, according to the minds and behavior of people on the threat of disease, it just needs to enhance the strength of treatment. In this case, the densities of susceptible, infected, and recovered individuals turn very suddenly to a great degree to $S\left(t_{j}^{+}\left(H_{h}\right)\right)$, $\left(1-q_{2}\right) I\left(t_{j}^{+}\left(H_{l}\right)\right)$, and $R\left(t_{j}^{+}\left(H_{h}\right)\right)+q_{2} I\left(t_{j}^{+}\left(H_{l}\right)\right)$, respectively, where $q_{2} \in(0,1)$ is the medication intensification effort.

Under the above assumptions, it follows from model (1) that we propose the following multiple state dependent pulse control differential equations:

(i) $I \neq H_{h}, H_{l}$, or $I=H_{h}, S<S_{1}^{*}$, or $I=H_{l}, S<S_{2}^{*}$ :

$$
\begin{aligned}
& \frac{\mathrm{d} S(t)}{\mathrm{d} t}=\mu-\beta \exp (-\alpha I) S I-\mu S, \\
& \frac{\mathrm{d} I(t)}{\mathrm{d} t}=\beta \exp (-\alpha I) S I-(\mu+\gamma) I, \\
& \frac{\mathrm{d} R(t)}{\mathrm{d} t}=\gamma I-\mu R ;
\end{aligned}
$$

(ii) $I=H_{h}$ and $S \geq S_{1}^{*}$ :

$$
\begin{aligned}
\Delta S(t) & =-p S(t), \\
\Delta I(t) & =-q_{1} I(t), \\
\Delta R(t) & =p S(t)+q_{1} I(t) ;
\end{aligned}
$$

(iii) $I=H_{l}$ and $S \geq S_{2}^{*}$ :

$$
\begin{aligned}
& \Delta S(t)=0, \\
& \Delta I(t)=-q_{2} I(t), \\
& \Delta R(t)=q_{2} I(t),
\end{aligned}
$$

where $S_{1}^{*}>0$ and $S_{2}^{*}>0$ are the abscissa of intersection of the the horizontal isocline $\beta \exp (-\alpha I) S-\mu-\gamma=0$ and the lines $I=H_{h}$ and $I=H_{l}$, respectively, and $S_{1}^{*}=(\mu+\gamma) \exp \left(\alpha H_{h}\right) / \beta$ and $S_{2}^{*}=(\mu+\gamma) \exp \left(\alpha H_{l}\right) / \beta$.

Let $\mathbb{R}=(-\infty, \infty)$ and $\mathbb{R}_{+}^{3}=\{(x, y, z) \mid x>0, y>$ $0, z>0\}$. The global existence and uniqueness of solution for systems (4)-(6) are guaranteed by the smoothness of the right-hand sides of systems (4)-(6). For more details, we refer to [22].

On the positive and ultimate boundedness of solutions of systems (4)-(6), we introduce the following Lemma 1.
Lemma 1. For any $p \in(0,1), q_{i} \in(0,1)(i=1,2)$, and each component of the solution $(S(t), I(t), R(t))$ of systems (4)(6) with initial value $(S(0), I(0), R(0)) \in \mathbb{R}_{+}^{3}$ is positive and ultimately bounded for all $t \in[0,+\infty)$.

The proof of Lemma 1 is similar to Lemma 1 in [11]; hence we omit it here.

Since from systems (4)-(6), without loss of generality, the total population is normalized to unity that $S(t)+I(t)+R(t)=$ 1 , therefore, systems (4)-(6) are equivalent to the following system:

$$
\begin{aligned}
\frac{\mathrm{d} S(t)}{\mathrm{d} t} & =\mu-\beta \exp (-\alpha I) S I-\mu S, \\
\frac{\mathrm{d} I(t)}{\mathrm{d} t} & =\beta \exp (-\alpha I) S I-(\mu+\gamma) I, \\
I & \neq H_{h}, H_{l} \text { or } I=H_{h}, S<S_{1}^{*} \text { or } I=H_{l}, S<S_{2}^{*}, \\
\Delta S(t) & =-p S(t), \\
\Delta I(t) & =-q_{1} I(t), \quad I=H_{h}, S \geq S_{1}^{*}, \\
\Delta S(t) & =0, \\
\Delta I(t) & =-q_{2} I(t), \\
S(0) & =S_{0}>0, \\
I(0) & =I_{0}>0 .
\end{aligned}
$$

Based on the biological background of model (7), we only consider dynamical behavior of model (7) in region $\Omega=$ $\{(S, I): S>0, I>0, S+I \leq 1\}$.

Generally, a semidynamical system $\left(X, \pi, \mathbb{R}_{+}\right)$is denoted by $(X, \pi)$. For any $P \in X$, the function $\pi_{P}: \mathbb{R}_{+} \rightarrow X$ defined as $\pi_{P}(t)=\pi(P, t)$ is continuous, and we call $\pi_{P}(t)$ the trajectory passing through point $P$. Consider the following general state dependent pulse differential equation:

$$
\begin{array}{ll}
\frac{\mathrm{d} x}{\mathrm{~d} t}=f(x, y), & \\
\frac{\mathrm{d} y}{\mathrm{~d} t}=g(x, y), & \varphi(x, y) \neq 0, \\
\Delta x=\alpha(x, y), & \varphi(x, y)=0,
\end{array}
$$

where $(x, y) \in \mathbb{R}^{2} . f, g, \alpha$, and $\beta$ are continuous functions mapping $\mathbb{R}^{2}$ into $\mathbb{R}$ and $\mathscr{M} \subset \mathbb{R}^{2}$ is the set of impulses. According to the denotations in [22], we denote $\mathscr{N}=\mathscr{I}(\mathscr{M})$, for any $P \in \mathscr{M}, \mathscr{I}(P)=P^{+} \in \mathcal{N}$, where $\mathscr{M}=\{(x, y):(x, y) \in$ $\left.\mathbb{R}^{2}, \varphi(x, y)=0\right\}$ is the set of impulses, $\mathscr{I}$ is the pulse function, 
and $\mathcal{N}$ is the set of phase after impulses. Obviously, the solution mapping of system (8) is a semicontinuous dynamical system, which is denoted by $(X, \pi, \mathscr{M}, \mathscr{I})$. Obviously, in model (7), we discuss in the paper, which is a semicontinuous dynamical system. For the sake of investigating the existence and stability of periodic solution of model (7), we give the following definitions and lemmas.

Definition 2 (semicontinuous dynamical system [22]). A triple $\left(X, \pi, \mathbb{R}_{+}\right)$is said to be a semidynamical system if $X$ is a metric space, $\mathbb{R}_{+}$is the set of all nonnegative real, and $\pi(P, t): X \times \mathbb{R}_{+} \rightarrow X$ is a continuous map such that

(a) $\pi(P, 0)=P$ for all $P \in X$,

(b) $\pi(\pi(P, t), s)=\pi(P, t+s)$ for all $P \in X$ and $t, s \in \mathbb{R}_{+}$.

Definition 3 (order-1 periodic solution [23]). A trajectory $\pi\left(P_{0}, t\right)$ is called order-1 periodic solution with period $T$ if there exists a point $P_{0} \in \mathscr{M}$ and $T>0$ such that $\pi\left(P_{0}, t\right)=P \in \mathscr{M}$ and $\mathscr{I}=\mathscr{I}\left(\pi\left(P_{0}, T\right)\right)=P_{0} \in \mathscr{N}$.

Definition 4 (orbitally asymptotically stable [23]). Suppose $\pi\left(P_{0}, t\right)$ is an order-1 periodic solution of model (8). If, for any $\varepsilon>0$, there must exist $\delta>0$ and $t_{0} \geq 0$, such that for any point $P_{1} \in U\left(P_{0}, \delta\right) \cap \mathcal{N}$, one has $d\left(\pi\left(P_{1}, t\right), \pi\left(P_{0}, t\right)\right)<\varepsilon$ for $t>t_{0}$, where $U\left(P_{0}, \delta\right)$ denotes a $\delta$-neighborhood of point $P_{0} \in \mathcal{N}$ and $d\left(\pi\left(P_{1}, t\right), \pi\left(P_{0}, t\right)\right)$ is the distance from $\pi\left(P_{1}, t\right)$ to $\pi\left(P_{0}, t\right)$. Then one calls the order-1 periodic solution $\pi\left(P_{0}, t\right)$ orbitally asymptotically stable.
Definition 5 (successor function [23]). Suppose $\phi: \mathcal{N} \rightarrow \mathcal{N}$ is a map. For any $P\left(x_{P}, y_{P}\right) \in \mathcal{N}$, if there exists a $t_{1}>0$ such that $\pi\left(P, t_{1}\right)=P_{1}\left(x_{P_{1}}, y_{P_{1}}\right) \in \mathscr{M}, P_{1}^{+}\left(x_{P_{1}^{+}}, y_{P_{1}^{+}}\right)=\mathscr{I}\left(P_{1}\right) \in \mathscr{N}$, then $\phi(P)=x_{P_{1}^{+}}-x_{P}$ is called the successor function of point $P$, and the point $P_{1}^{+}$is called the successor point of $P$.

The following lemma and remarks are on the properties of successor function $\phi(P)$.

Lemma 6. The successor function $\phi(P)$ is continuous.

The proof of Lemma 6 is obvious; hence we omit it here.

Remark 7. From Lemma 6, it is obvious that model (8) exists as positive order-1 periodic solution if there exist two points $P_{1}, P_{2} \in \mathcal{N}$ satisfying $\phi\left(P_{1}\right) \phi\left(P_{2}\right)<0$.

Remark 8. In Lemma 6, if $\phi(P)=0$, then trajectory $\pi\left(P_{0}, t\right)$ with initial point $P_{0}$ is an order-1 periodic solution of model (8).

The following Lemma 9 is on the orbitally asymptotical stability of periodic solution of model (8), which comes from Corollary 2 of Theorem 1 of [24].

Lemma 9 (analogue of Poincaré Criterion [24]). The Tperiodic solution $(\phi(t), \psi(t))$ of system (8) is orbitally asymptotically stable if the Floquet multiplier $\mu$ satisfies the condition $|\mu|<1$, where

$$
\begin{aligned}
\mu & =\prod_{j=1}^{n} \Delta_{j} \exp \left\{\int_{0}^{T}\left(\frac{\partial f(\phi(t), \psi(t))}{\partial x}+\frac{\partial g(\phi(t), \psi(t))}{\partial y}\right) \mathrm{d} t\right\} \\
\Delta_{j} & =\frac{((\partial \eta / \partial y)(\partial \varphi / \partial x)-(\partial \eta / \partial x)(\partial \varphi / \partial y)+\partial \varphi / \partial x) f_{+}+((\partial \xi / \partial x)(\partial \varphi / \partial y)-(\partial \xi / \partial y)(\partial \varphi / \partial x)+\partial \varphi / \partial y) g_{+}}{(\partial \varphi / \partial x) f+(\partial \varphi / \partial y) g}
\end{aligned}
$$

and $f, g, \partial \xi / \partial x, \partial \xi / \partial y, \partial \eta / \partial x, \partial \eta / \partial y, \partial \varphi / \partial x$, and $\partial \varphi / \partial y$ are calculated at the point $\left(\phi\left(\tau_{j}\right), \psi\left(\tau_{j}\right)\right), f_{+}=f\left(\phi\left(\tau_{j}^{+}\right), \psi\left(\tau_{j}^{+}\right)\right)$, $g_{+}=g\left(\phi\left(\tau_{j}^{+}\right), \psi\left(\tau_{j}^{+}\right)\right)$, and $\tau_{j}(j \in N)$ is the time of the $j$ th jump.

To discuss the dynamical behaviors of model (7), we denote two pulse sets

$$
\begin{aligned}
& \Sigma_{1}:=\left\{(S, I): S_{1}^{*} \leq S \leq 1-H_{h}, I=H_{h}\right\}, \\
& \Sigma_{2}:=\left\{(S, I): S_{2}^{*} \leq S \leq 1-H_{l}, I=H_{l}\right\}
\end{aligned}
$$

and two phase sets

$$
\begin{aligned}
\Sigma_{q_{1}} & :=\mathscr{I}_{1}\left(\Sigma_{1}\right)=\left\{(S, I):(1-p) S_{1}^{*} \leq S\right. \\
\leq & \left.(1-p)\left(1-H_{h}\right), I=\left(1-q_{1}\right) H_{h}\right\}, \\
\Sigma_{q_{2}} & :=\mathscr{I}_{2}\left(\Sigma_{2}\right)=\left\{(S, I): S_{2}^{*} \leq S \leq 1-H_{l}, I\right. \\
& \left.=\left(1-q_{2}\right) H_{l}\right\},
\end{aligned}
$$

where $\mathscr{I}_{1}:\left(S, H_{h}\right) \in \Sigma_{1} \rightarrow\left((1-p) S,\left(1-q_{1}\right) H_{h}\right) \in \Omega$ and $\mathscr{I}_{2}:\left(S, H_{l}\right) \in \Sigma_{2} \rightarrow\left(S,\left(1-q_{2}\right) H_{l}\right) \in \Omega$ are continuous functions.

\section{Main Results}

Since the endemic equilibrium $E^{*}\left(S^{*}, I^{*}\right)$ of model (7) without pulse effect is globally asymptotically stable, then any positive solutions of model (7) without pulse effect will eventually tend to $E^{*}$. Therefore, region $\Omega$ is divided into four different domains with the vertical isocline $\mathrm{d} S / \mathrm{d} t=0$ and the horizontal isocline $\mathrm{d} I / \mathrm{d} t=0$ of model (7), where

$$
\begin{aligned}
& \mathbb{D}_{1}:=\left\{(S, I) \in \Omega: \frac{\mathrm{d} S}{\mathrm{~d} t}>0, \frac{\mathrm{d} I}{\mathrm{~d} t}<0\right\}, \\
& \mathbb{D}_{2}:=\left\{(S, I) \in \Omega: \frac{\mathrm{d} S}{\mathrm{~d} t}>0, \frac{\mathrm{d} I}{\mathrm{~d} t}>0\right\},
\end{aligned}
$$




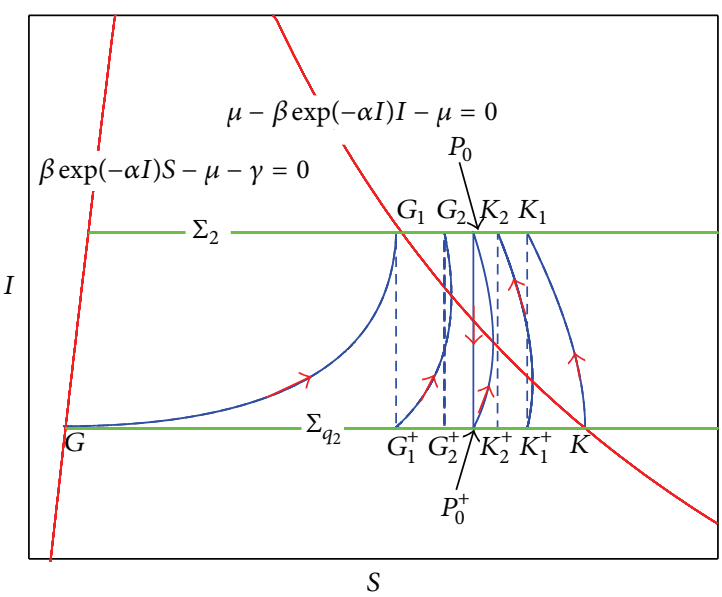

(a)

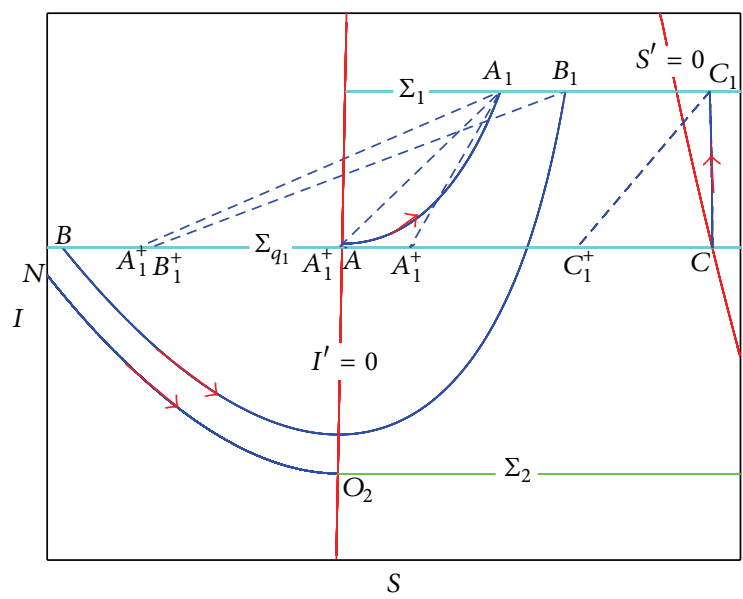

(b)

Figure 2: (a) The illustration on the existence of order-1 periodic solution of model (7) starting from pulse set $\Sigma_{q_{2}}$; (b) The illustration of existence of order-1 periodic solution of model (7) starting from pulse set $\Sigma_{q_{1}}$.

$$
\begin{aligned}
& \mathbb{D}_{3}:=\left\{(S, I) \in \Omega: \frac{\mathrm{d} S}{\mathrm{~d} t}<0, \frac{\mathrm{d} I}{\mathrm{~d} t}>0\right\}, \\
& \mathbb{D}_{4}:=\left\{(S, I) \in \Omega: \frac{\mathrm{d} S}{\mathrm{~d} t}<0, \frac{\mathrm{d} I}{\mathrm{~d} t}<0\right\} .
\end{aligned}
$$

For convenience, we denote the $I$-axis intersect line $I=$ $H_{l}$ at point $M\left(0, H_{l}\right)$. Suppose that the horizontal isocline line $\beta \exp (-\alpha I) S-\mu-\gamma=0$ intersects lines $I=H_{h}, I=\left(1-q_{1}\right) H_{h}$, and $I=\left(1-q_{2}\right) H_{l}$ at points $O_{1}\left(S_{1}^{*}, H_{h}\right), A\left(S_{A},\left(1-q_{1}\right) H_{h}\right)$, and $G\left(S_{G},\left(1-q_{2}\right) H_{l}\right)$, respectively. Suppose that the vertical isocline line $\mu-\beta \exp (-\alpha I) S-\mu S=0$ intersects lines $I=$ $\left(1-q_{1}\right) H_{h}$ and $I=\left(1-q_{2}\right) H_{l}$ at points $C\left(S_{C},\left(1-q_{1}\right) H_{h}\right)$ and $K\left(S_{K},\left(1-q_{2}\right) H_{l}\right)$, respectively.

According to the uniqueness of solution to initial value, we know there exists a unique trajectory $\pi_{N}(t)$ starting from the initial point $N\left(0, I_{N}\right)\left(I_{N}>H_{l}\right)$ and tangent to line $I=H_{l}$ at point $\mathrm{O}_{2}=\left(S_{2}^{*}, H_{l}\right)$. Let

$$
\begin{aligned}
& \Omega_{1}=\left\{(S, I) \in \Omega: H_{l}<I \leq H_{h}\right\}, \\
& \Omega_{2}=\left\{(S, I) \in \Omega: I \leq H_{l}\right\}
\end{aligned}
$$

and let $\Omega_{3}$ be a bounded domain by the phase trajectory $\overparen{\mathrm{NO}_{2}}$ and segments $\overline{\mathrm{O}_{2} M}$ and $\overline{M N}$. Obviously, if $H_{h}<I^{*}$, then any trajectory with initial value $\left(S_{0}, I_{0}\right) \in \Omega_{1} / \Omega_{3}$ will reach pulse set $\Sigma_{1}$, and any trajectory with initial value $\left(S_{0}, I_{0}\right) \in \Omega_{2} \cup$ $\Omega_{3}$ will reach pulse set $\Sigma_{2}$. So, in this section, we discuss the existence and stability of positive order-1 periodic solutions of model (7) in cases of initial values $\left(S_{0}, I_{0}\right) \in \Omega_{1} / \Omega_{3}$ and $\left(S_{0}, I_{0}\right) \in \Omega_{2} \cup \Omega_{3}$, respectively.

Firstly, the following result is on the existence and stability of positive order-1 periodic solution for model (7).
Theorem 10. For any $p \in(0,1), q_{1} \in(0,1)$, and $q_{2} \in(0,1)$, model (7) has always an orbitally asymptotically stable positive order-1 periodic solution and which starts from pulse set $\Sigma_{q_{2}}$.

Proof. Obviously, any trajectory with initial value $\left(S_{0}, I_{0}\right) \in$ $\Omega_{2} \cup \Omega_{3}$ will reach pulse set $\Sigma_{2}$ and intersects pulse set $\Sigma_{2}$ infinite times due to pulse treatment $\Delta I(t)=I\left(t^{+}\right)-I(t)=$ $-q_{2} I(t)$. The trajectory $\pi_{G}(t)$ passing through point $G$ which is tangent to phase set $\Sigma_{q_{2}}$ at point $G$ intersects with pulse set $\Sigma_{2}$ at point $G_{1}\left(S_{G_{1}}, H_{l}\right)$ and then jumps to point $G_{1}^{+}\left(S_{G_{1}^{+}},(1-\right.$ $\left.q_{2}\right) H_{l}$ ) due to pulse treatment $\Delta I(t)=I\left(t^{+}\right)-I(t)=-q_{2} I(t)$, where $S_{G_{1}^{+}}=S_{G_{1}}$. By the geometrical construction of phase region $\mathbb{D}_{2}$, we have that point $G_{1}^{+}$is right point $G$. That is, $S_{G}<$ $S_{G_{1}^{+}}$. Hence the successor function of point $G$ is $\phi(G)=S_{G_{1}^{+}}$ $S_{G}>0$.

On the other hand, trajectory $\pi_{K}(t)$ from the initial point $N\left(S_{K},\left(1-q_{2}\right) H_{l}\right)$ intersects pulse set $\Sigma_{2}$ at point $K_{1}\left(S_{K_{1}}, H_{l}\right)$ and next jumps to point $K_{1}^{+}\left(S_{K_{1}^{+}},\left(1-q_{2}\right) H_{l}\right)\left(S_{K_{1}^{+}}=S_{K_{1}}\right)$ on phase set $\Sigma_{q_{2}}$. It follows from the geometrical construction of region $\mathbb{D}_{3}$ that we have that point $K_{1}^{+}$is right point $K$. That is, $S_{K_{1}^{+}}<S_{K}$. Therefore, we have $\phi(K)=S_{K_{1}^{+}}-S_{K}<0$.

By Lemma 6, we know that there exists a positive order-1 periodic solution of model (7), which starts from pulse set $\Sigma_{q_{2}}$ (for more details, see Figure 2(a)).

Next, we discuss the orbital stability of positive order1 periodic solution which starts from pulse set $\Sigma_{q_{2}}$. Since trajectories starting from any point $(S, I) \in \Omega_{1} \cup \Omega_{3}$ will enter the set $\overline{G_{1} K_{1}}=\left\{(S, I) \in \Sigma_{q_{2}} \mid S_{G_{1}} \leq S \leq S_{K_{1}}\right\}$ after several times pulse effects at most, then the initial point of the order1 periodic solution only lies in $\overline{G_{1}^{+} K_{1}^{+}}$. The set $\overline{G_{1}^{+} K_{1}^{+}}$is mapped to set $\overline{G_{2} K_{2}}=\left\{(S, I) \in \Sigma_{1} \mid S_{G_{2}} \leq S \leq S_{K_{2}}\right\}$ by the geometrical construction of the phase regions $\mathbb{D}_{2}$ and $\mathbb{D}_{3}$. Subsequently, set $\overline{G_{2} K_{2}}$ is mapped to set $\overline{G_{2}^{+} K_{2}^{+}}=\left\{(S, I) \in \Sigma_{q_{2}} \mid S_{G_{2}^{+}} \leq S \leq\right.$ $S_{K_{2}^{+}}$d due to the pulse vaccination and pulse treatment. From the geometrical construction of regions $\mathbb{D}_{2}$ and $\mathbb{D}_{3}$, it is easy 
to know that $\left|\overline{G_{1}^{+} K_{1}^{+}}\right|>\left|\overline{G_{2}^{+} K_{2}^{+}}\right|$. Repeating the abovementioned process, we can get two sequences $\left\{G_{n}^{+}\left(S_{G_{n}^{+}},\left(1-q_{2}\right) H_{l}\right)\right\}$ and $\left\{K_{n}^{+}\left(S_{G_{n}^{+}},\left(1-q_{2}\right) H_{l}\right)\right\}(n=1,2, \ldots)$ on set $\Sigma_{q_{2}}$ and satisfy

$$
\begin{gathered}
S_{G}<S_{G_{1}^{+}}<\cdots<S_{G_{n}^{+}}<\cdots<S_{K_{n}^{+}}<S_{K_{1}^{+}}<S_{K}, \\
|\overline{G K}|>\left|\overline{G_{1}^{+} K_{1}^{+}}\right|>\cdots>\left|\overline{G_{n}^{+} K_{n}^{+}}\right|>\cdots .
\end{gathered}
$$

Therefore, the sequence $\left\{\left|\overline{G_{n}^{+} K_{n}^{+}}\right|\right\}$is convergent monotonously and $\lim _{n \rightarrow \infty}\left|\overline{G_{n}^{+} K_{n}^{+}}\right|=0$. It implies that there exists a unique point $P_{0}^{+} \in\left[S_{G_{n}^{+}}, S_{K_{1}^{+}}\right]$such that $g\left(P_{0}^{+}\right)=0$. Furthermore, we have $\lim _{n \rightarrow \infty} S_{G_{n}^{+}}=\lim _{n \rightarrow \infty} S_{K_{n}^{+}}=S_{P_{0}^{+}}$. For any point $Q\left(S_{Q},\left(1-q_{2}\right) H_{l}\right) \in \Sigma_{q_{2}}$, where

$$
\begin{aligned}
& S_{Q} \in {\left[S_{G}, S_{K_{1}^{+}}\right) \cup\left[S_{G_{n}^{+}}, S_{G_{n+1}^{+}}\right) \cup\left[S_{K_{n+1}^{+}}, S_{K_{n}^{+}}\right) } \\
& \cup\left[S_{K_{1}^{+}}, S_{K^{+}}\right), \quad n=1,2, \ldots
\end{aligned}
$$

Without loss of generality, let $S_{\mathrm{Q}} \in\left[S_{G_{n}^{+}}, S_{G_{n+1}^{+}}\right)$, the trajectory $\pi_{\mathrm{Q}}(t)$ starting from the initial point $Q\left(S_{Q},\left(1-q_{2}\right) H_{l}\right) \in \Sigma_{q_{2}}$ intersects pulse set $\Sigma_{2}$ at point $Q_{1}\left(S_{Q_{1}}, H_{l}\right)\left(S_{Q_{1}} \in\left[S_{G_{n+1}}, S_{G_{n+2}}\right)\right)$ and next jumps to point $Q_{1}^{+}\left(S_{Q_{1}^{+}},\left(1-q_{2}\right) H_{l}\right) \in \Sigma_{q_{2}}$ due to pulse vaccination and pulse treatment, where $S_{\mathrm{Q}_{1}^{+}} \in\left[S_{G_{n+1}^{+}}, S_{G_{n+2}^{+}}\right)$. Repeating the process, we obtain a sequence $\left\{Q_{n}^{+}\left(S_{Q_{n}^{+}},\left(1-q_{2}\right) H_{l}\right)\right\}$ of set $\Sigma_{q_{2}}$, where $S_{Q_{n}^{+}} \in\left[S_{G_{n+k+1}^{+}}, S_{G_{n+k}^{+}}\right), k=1,2, \ldots$. Since $\lim _{n \rightarrow \infty} S_{G_{n}^{+}}=S_{P_{0}^{+}}$, then $\lim _{n \rightarrow \infty} S_{Q_{n}^{+}}=S_{P_{0}^{+}}$. That is, $\lim _{n \rightarrow \infty} Q_{n}^{+}=P_{0}^{+}$. Similarly, if $S_{Q} \in\left[S_{K_{n+1}^{+}}, S_{K_{n}^{+}}\right)$, we also can get $\lim _{n \rightarrow \infty} Q_{n}^{+}=P_{0}^{+}$. Thus trajectory starts from any point $Q$ of set $\Sigma_{q_{2}}$, which ultimately tends to the positive order-1 periodic solution $\widehat{P_{0}^{+} P_{0} P_{0}^{+}}$.

Given the above, we obtain that model (7) has a positive order-1 periodic solution, which starts from the initial value $\left(S_{0}, I_{0}\right) \in \Omega_{2} \cup \Omega_{3}$ and is orbitally asymptotically stable. This completes the proof.

Theorem 11. If $I_{N} \leq\left(1-q_{1}\right) H_{h}$, for any $p \in(0,1), q_{1} \in(0,1)$, and $q_{2} \in(0,1)$, then model (7) has two positive order-1 periodic solutions in region $\Omega_{1} \cup \Omega_{2}$ : one starts from pulse set $\Sigma_{q_{1}}$, and the other starts from $\Sigma_{q_{2}}$. Furthermore, let $(\phi(t), \psi(t))$ be an order-1 periodic solution which starts from the pulse set $\Sigma_{q_{1}}$ of model (7) with period T; if

$$
\begin{aligned}
|\mu| & =\left|\frac{(1-p)\left[\beta e^{-\alpha\left(1-q_{1}\right) H_{h}}(1-p) \phi(T)-\mu-\gamma\right]}{\beta e^{-\alpha H_{h}} \phi(T)-\mu-\gamma}\right| \\
\cdot & \exp \left\{-\int_{0}^{T}\left[\mu+(1+\alpha \phi(t)) \beta e^{-\alpha \psi(t)} \psi(t)\right] \mathrm{d} t\right\} \\
& <1,
\end{aligned}
$$

then $(\phi(t), \psi(t))$ is orbitally asymptotically stable. Thus, trajectory of model (7) with initial value $\left(S_{0}, I_{0}\right) \in \Omega_{1} \cup \Omega_{3}$ will tend to the stable positive order-1 periodic solution which starts from pulse set $\Sigma_{q_{2}}$, and trajectory of model (7) with initial value $\left(S_{0}, I_{0}\right) \in \Omega_{1} / \Omega_{3}$ will tend to the stable positive order-1 periodic solution which starts from $\Sigma_{q_{1}}$.
Proof. The proof of the existence and stability of the positive order-1 periodic solution starting from pulse set $\Sigma_{q_{2}}$ has appeared in Theorem 10. We just need to prove the existence and stability of the other positive order-1 periodic solution which starts from pulse set $\Sigma_{q_{1}}$.

Suppose that the trajectory passing through point $A\left(S_{A},\left(1-q_{1}\right) H_{h}\right)$ intersects with pulse set $\Sigma_{1}$ at point $A_{1}\left(S_{A_{1}}, H_{h}\right)$. Since point $A_{1} \in \Sigma_{1}$, then pulse occurs at $A_{1}$; supposing point $A_{1}$ is subject to pulse effects to point $A_{1}^{+}\left(S_{A_{1}^{+}},\left(1-q_{1}\right) H_{h}\right)$, where $S_{A_{1}^{+}}=(1-p) S_{A_{1}}$, the position of $A_{1}^{+}$has the following three cases (for more details, see Figure 2(b)):

(i) If point $A_{1}^{+}$coincides with $A$, that is, $S_{A}=S_{A_{1}^{+}}$, then the successor function of $A$ is that $f(A)=S_{A_{1}^{+}}$$S_{A}=0$. Thus, model (7) has a positive order-1 periodic solution which starts from pulse set $\Sigma_{q_{1}}$.

(ii) If point $A_{1}^{+}$is left point $A$, that is, $S_{A}>S_{A_{1}^{+}}$, thus the successor function of $A$ is that $f(A)=S_{A_{1}^{+}}-$ $S_{A}<0$. On the other hand, choose a point $B(\varepsilon,(1-$ $\left.\left.q_{1}\right) H_{h}\right) \in \Sigma_{q_{1}}$ which is left point $A_{1}^{+}$, where $\varepsilon \in$ $\left(0,(1-p) S_{1}^{*}\right)$ is small enough. Suppose that trajectory from the initial point $B\left(\varepsilon,\left(1-q_{1}\right) H_{h}\right)$ intersects pulse set $\Sigma_{1}$ at point $B_{1}\left(S_{B_{1}}, H_{h}\right)$ and next jumps to point $B_{1}^{+}\left(S_{B_{1}^{+}},\left(1-q_{1}\right) H_{h}\right)$ on phase set $\Sigma_{q_{1}}$ due to pulse effects. According to the existence and uniqueness of solution for pulse differential equation, point $B_{1}$ is right point $A_{1}$ and point $B_{1}^{+}$is right point $A_{1}^{+}$. Therefore, the successor function of point $B$ is $f(B)=$ $S_{B_{1}^{+}}-S_{B}>0$. By Lemma 6, we know that there exists a positive order-1 periodic solution of model (7), which starts from pulse set $\Sigma_{q_{1}}$.

(iii) If point $A_{1}^{+}$is right point $A$, that is, $S_{A}<S_{A_{1}^{+}}$, thus, the successor function of $A$ is that $f(A)=S_{A_{1}^{+}}-S_{A}>$ 0 . In the meanwhile, suppose that trajectory from the initial point $C\left(S_{C},\left(1-q_{1}\right) H_{h}\right)$ intersects pulse set $\Sigma_{1}$ at point $C_{1}\left(S_{C_{1}}, H_{h}\right)$ and next jumps to point $C_{1}^{+}\left(S_{C_{1}^{+}},\left(1-q_{1}\right) H_{h}\right)$ on phase set $\Sigma_{q_{1}}$ again, where $S_{C_{1}^{+}}=(1-P) S_{C_{1}}$. From the geometrical construction of phase region $\mathbb{D}_{3}$ of model (7), we have $S_{C_{1}}<S_{C}$. Therefore, point $C_{1}^{+}$is left point $C$. That is, $S_{C_{1}^{+}}<S_{C}$. Thus, the successor function of $A$ is $f(C)=S_{C_{1}^{+}}-S_{C}<$ 0 . By Lemma 6, we know that model (7) admits a positive order-1 periodic solution, which starts from pulse set $\Sigma_{q_{1}}$.

To sum up the above discussion, model (7) exists as an order-1 periodic solution $(\phi(t), \psi(t))$ which starts from pulse set $\Sigma_{q_{1}}$.

Next, we show the orbitally asymptotical stability of this order-1 periodic solution $(\phi(t), \psi(t))$. According to Lemma 9, suppose that $(\phi(t), \psi(t))$ intersects phase set $\Sigma_{\mathrm{q}_{1}}$ and pulse set 
$\Sigma_{1}$ at points $P^{+}\left((1-p) \phi(T),\left(1-q_{1}\right) H_{h}\right)$ and $P\left(\phi(T), H_{h}\right)$, respectively. Comparing with model (8), we have

$$
\begin{aligned}
& f(S, I)=\mu-\beta e^{-\alpha I} S I-\mu S, \\
& g(S, I)=\beta e^{-\alpha I} S I-(\mu+\gamma) I,
\end{aligned}
$$

$\xi(S, I)=-p S, \eta(x, y)=-q_{1} H_{h}$, and $\varphi(S, I)=I-H_{h}$. Thus,

$$
\begin{aligned}
& \frac{\partial f}{\partial S}=-\beta e^{-\alpha I} I-\mu, \\
& \frac{\partial g}{\partial I}=-\alpha \beta e^{-\alpha I} S I+\beta e^{-\alpha I} S-\mu-\gamma,
\end{aligned}
$$

$$
\begin{aligned}
& \frac{\partial \xi}{\partial S}=-p, \\
& \frac{\partial \eta}{\partial I}=-q_{1}, \\
& \frac{\partial \varphi}{\partial I}=1, \\
& \frac{\partial \xi}{\partial I}=\frac{\partial \eta}{\partial S}=\frac{\partial \varphi}{\partial S}=0 .
\end{aligned}
$$

Furthermore, it follows that

$$
\begin{aligned}
\Delta_{1} & =\frac{((\partial \eta / \partial I)(\partial \varphi / \partial S)-(\partial \eta / \partial S)(\partial \varphi / \partial I)+\partial \varphi / \partial S) f_{+}+((\partial \xi / \partial S)(\partial \varphi / \partial I)-(\partial \xi / \partial I)(\partial \varphi / \partial S)+\partial \varphi / \partial I) g_{+}}{(\partial \varphi / \partial S) f+(\partial \varphi / \partial I) g} \\
& =\frac{(1-p) g_{+}\left(\phi\left(T^{+}\right), \psi_{2}\left(T^{+}\right)\right)}{g\left(\phi_{1}(T), \psi_{2}(T)\right)}=\frac{(1-p)\left(1-q_{1}\right)\left[\beta e^{-\alpha\left(1-q_{1}\right) H_{h}}(1-p) \phi(T)-\mu-\gamma\right]}{\beta e^{-\alpha H_{h}} \phi(T)-\mu-\gamma}, \\
\mu & =\Delta_{1} \exp \left\{\int_{0}^{T}\left[\frac{\partial f}{\partial S}(\phi(t), \psi(t))+\frac{\partial g}{\partial I}(\phi(t), \psi(t))\right] \mathrm{d} t\right\} \\
& =\Delta_{1} \exp \left\{\int_{0}^{T}\left[-\beta e^{-\alpha \psi(t)} \psi(t)-\mu-\alpha \beta e^{-\alpha \psi(t)} \phi(t) \psi(t)+\beta e^{-\alpha \psi(t)} \phi(t)-\mu-\gamma\right] \mathrm{d} t\right\} .
\end{aligned}
$$

On the other hand, we integrate both sides of the second equation of model (7) along the orbit $\overparen{P^{+} P}$, and we have

$$
\begin{aligned}
\ln \frac{1}{1-q_{1}} & =\int_{\left(1-q_{1}\right) H_{h}}^{H_{h}} \frac{\mathrm{d} I}{I} \\
& =\int_{0}^{T}\left[\beta e^{-\alpha \psi(t)} \phi(t)-\mu-\gamma\right] \mathrm{d} t .
\end{aligned}
$$

Then, we have

$$
\begin{aligned}
& |\mu| \\
& =\frac{1}{1-q_{1}}\left|\frac{(1-p)\left(1-q_{1}\right)\left[\beta e^{-\alpha\left(1-q_{1}\right) H_{h}}(1-p) \phi(T)-\mu-\gamma\right]}{\beta e^{-\alpha H_{h}} \phi(T)-\mu-\gamma}\right| \\
& \cdot \exp \left\{-\int_{0}^{T}\left[\mu+(1+\alpha \phi(t)) \beta e^{-\alpha \psi(t)} \psi(t)\right] \mathrm{d} t\right\} \\
& =\left|\frac{(1-p)\left[\beta e^{-\alpha\left(1-q_{1}\right) H_{h}}(1-p) \phi(T)-\mu-\gamma\right]}{\beta e^{-\alpha H_{h}} \phi(T)-\mu-\gamma}\right| \\
& \cdot \exp \left\{-\int_{0}^{T}\left[\mu+(1+\alpha \phi(t)) \beta e^{-\alpha \psi(t)} \psi(t)\right] \mathrm{d} t\right\} .
\end{aligned}
$$

This shows that

$$
\begin{aligned}
& \exp \left\{-\int_{0}^{T}\left[\mu+(1+\alpha \phi(t)) \beta e^{-\alpha \psi(t)} \psi(t)\right] \mathrm{d} t\right\} \\
& <\exp (-\mu T) \leq \frac{1-\phi(T)}{1-(1-p) \phi(T)}
\end{aligned}
$$

The following Corollary 12 is a direct consequence of Theorem 11. 
Corollary 12. Let $(\phi(t), \psi(t))$ be the order-1 periodic solution starting from pulse set $\Sigma_{q_{1}}$ of model (7) with period T. If

$$
\begin{aligned}
|\mu| & =\left|\frac{(1-p)\left[\beta e^{-\alpha\left(1-q_{1}\right) H_{h}}(1-p) \phi(T)-\mu-\gamma\right]}{\beta e^{-\alpha H_{h}} \phi(T)-\mu-\gamma}\right| \\
\cdot & \frac{1-\phi(T)}{1-(1-p) \phi(T)}<1,
\end{aligned}
$$

then $(\phi(t), \psi(t))$ is orbitally asymptotically stable.

For $I_{N}>\left(1-q_{1}\right) H_{h}$, in this case, suppose that orbit $\widetilde{N_{2}}$ starting from the initial point $N\left(0, I_{N}\right)$ will intersect lines $I=\left(1-q_{1}\right) H_{h}$ and $I=H_{l}$ at points $D\left(S_{1},\left(1-q_{1}\right) H_{h}\right)$ and $\mathrm{O}_{2}\left(\mathrm{~S}_{2}^{*}, \mathrm{H}_{l}\right)$, respectively.

Integrating both sides of the second equation of model (7) along orbit $\widetilde{\mathrm{NO}_{2}}$, we have

$$
\begin{aligned}
\ln \frac{H_{l}}{\left(1-q_{1}\right) H_{h}} & =\int_{\left(1-q_{1}\right) H_{h}}^{H_{l}} \frac{\mathrm{d} I}{I} \\
& =\int_{t_{1}}^{t_{2}}\left(\beta e^{-\alpha I} S-\mu-\gamma\right) \mathrm{d} t \\
& >-(\mu+\gamma)\left(t_{2}-t_{1}\right) .
\end{aligned}
$$

That is,

$$
t_{2}-t_{1}>\frac{1}{\mu+\gamma} \ln \frac{\left(1-q_{1}\right) H_{h}}{H_{l}} .
$$

Further, integrating both sides of the first equation of model (7) along orbit $\widetilde{\mathrm{NO}_{2}}$, we have

$$
\begin{aligned}
S_{1} & =S_{2}^{*}-\int_{t_{1}}^{t_{2}}\left(\mu-\beta e^{-\alpha I} S I-\mu S\right) \mathrm{d} t<S_{2}^{*} \\
& -\left[\mu-\beta e^{-\alpha\left(1-q_{1}\right) H_{h}} S_{2}^{*}\left(1-q_{1}\right) H_{h}-\mu S_{2}^{*}\right]\left(t_{2}-t_{1}\right) .
\end{aligned}
$$

From the geometrical construction of phase region $\mathbb{D}_{1}$, we have $\mu-\beta e^{-\alpha I} S I-\mu S>0$. Since the point $\left(S_{2}^{*},\left(1-q_{1}\right) H_{h}\right)$ in region $\mathbb{D}_{1}$, it yields that

$$
\mu-\beta e^{-\alpha\left(1-q_{1}\right) H_{h}} S_{2}^{*}\left(1-q_{1}\right) H_{h}-\mu S_{2}^{*}>0 .
$$

This together with (26) and (27) gives

$$
\begin{gathered}
S_{1}<S_{2}^{*}-\left(\mu-\beta e^{-\alpha\left(1-q_{1}\right) H_{h}} S_{2}^{*}\left(1-q_{1}\right) H_{h}-\mu S_{2}^{*}\right) \\
\cdot \frac{1}{\mu+\gamma} \ln \frac{\left(1-q_{1}\right) H_{h}}{H_{l}}:=\theta^{*} .
\end{gathered}
$$

Theorem 13. Assuming that $I_{N}>\left(1-q_{1}\right) H_{h}$, for any $p \in$ $(0,1), q_{1} \in(0,1)$, and $q_{2} \in(0,1)$, model (7) has a positive order-1 periodic solution starting from pulse set $\Sigma_{q_{2}}$ which is orbitally asymptotically stable. Furthermore, if

$$
0<p \leq 1-\frac{\beta}{\mu+\gamma} \theta^{*} e^{-\alpha H_{h}},
$$

then model (7) has a positive order-1 periodic solution starting from pulse set $\Sigma_{q_{1}}$. Let $(\phi(t), \psi(t))$ be the order-1 periodic solution of model (7) with period $T$, which starts from pulse set $\Sigma_{q_{1}}$. Further, if (16) holds, then $(\phi(t), \psi(t))$ is orbitally asymptotically stable. Thus, model (7) has two positive order1 periodic solutions in region $\Omega_{1} \cup \Omega_{2}$, which are orbitally asymptotically stable.

The proof of the existence and stability of positive order-1 periodic solution starting from pulse set $\Sigma_{q_{1}}$ of model (7) is similar to the proof of Theorem 11, here omitted.

\section{Numerical Simulations}

To illustrate the theoretical results and the feasibility of state dependent pulse control strategies, some simulations are presented. In systems (4)-(6), we fixed parameters $\mu=0.1$, $\beta=0.8, \alpha=0.5$, and $\gamma=0.04$. That is, we consider the following SIR epidemic model with state-dependent pulse vaccination and medication:

$$
\begin{aligned}
& \frac{\mathrm{d} S(t)}{\mathrm{d} t}=0.1-0.8 e^{-0.5 I} S I-0.1 S, \\
& \frac{\mathrm{d} I(t)}{\mathrm{d} t}=0.8 e^{-0.5 I} S I-(0.1+0.04) I,
\end{aligned}
$$

$$
\begin{aligned}
\frac{\mathrm{d} R(t)}{\mathrm{d} t} & =0.04 I-0.1 R, \\
I & \neq H_{h}, H_{l} \text { or } I=H_{h}, S<S_{1}^{*} \text { or } I=H_{l}, S<S_{2}^{*},
\end{aligned}
$$

$$
\begin{aligned}
\Delta S(t) & =-p S(t), \\
\Delta I(t) & =-q_{1} I(t), \\
\Delta R(t) & =p S(t)+q_{1} I(t),
\end{aligned}
$$

$$
I=H_{h}, S \geq S_{1}^{*},
$$

$$
\begin{aligned}
& \Delta S(t)=0, \\
& \Delta I(t)=-q_{2} I(t), \\
& \Delta R(t)=q_{2} I(t),
\end{aligned}
$$

$$
I=H_{l}, S \geq S_{2}^{*}
$$

By directly calculating, we have $R_{0}=\beta /(\mu+\gamma) \approx 5.7143>1$. It is easy to known that model (31) without pulse control has a unique globally asymptotically stable endemic equilibrium $E^{*}\left(S^{*}, I^{*}, R^{*}\right)=(0.2304,0.5497,0.2199)$.

Firstly, we choose control parameters $q_{1}=q_{2}=0.3$, $H_{h}=0.45<I_{*}=0.5497$, and $H_{l}=0.25<(1-$ $\left.q_{1}\right) H_{h}=0.315$, respectively. By calculating, we get $S_{1}^{*}=$ $(\mu+\gamma) e^{\alpha H_{h}} / \beta=0.2192$ and $S_{2}^{*}=(\mu+\gamma) \exp \left(\alpha H_{l}\right) / \beta=0.1983$. From numerical simulation, we know model (31) has a unique 


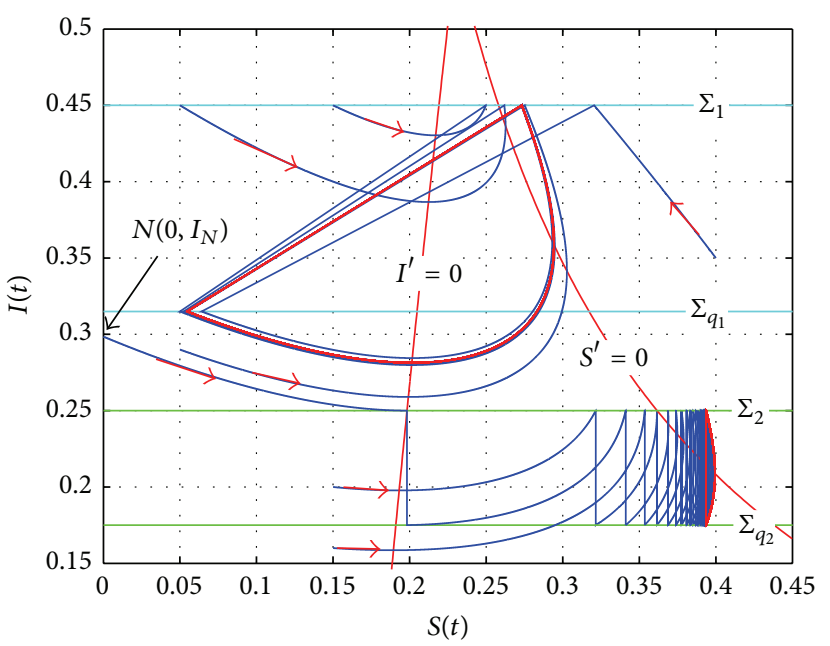

(a)

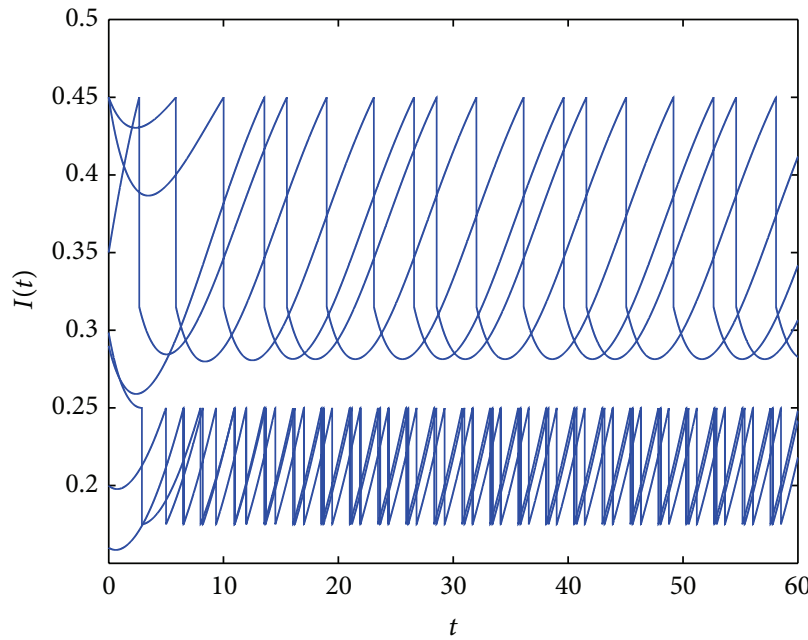

(c)

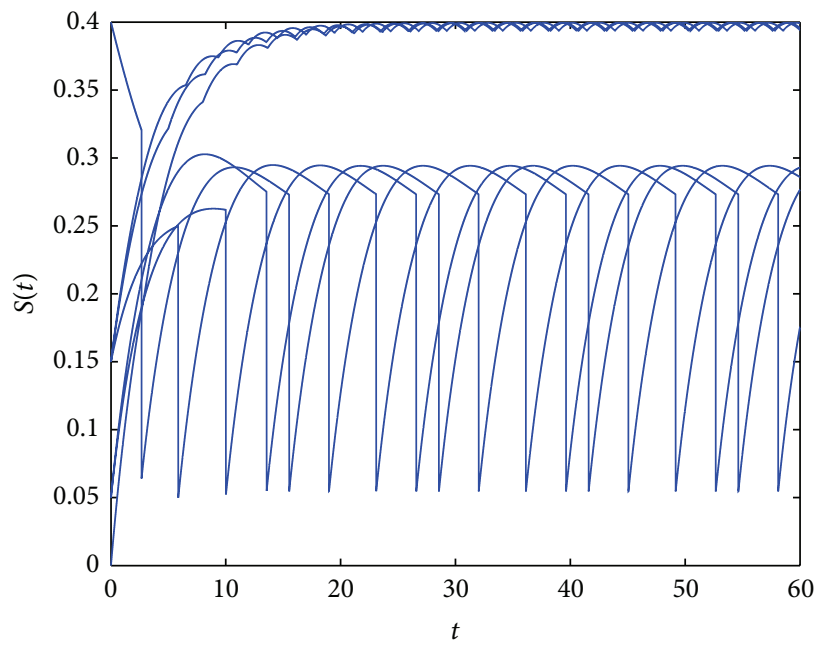

(b)

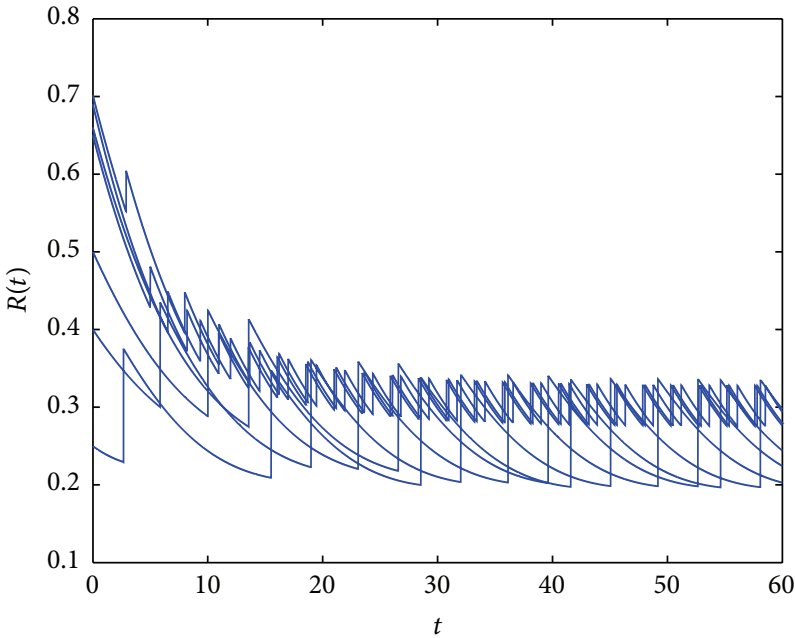

(d)

FIGURE 3: The existence and orbital stability of order-1 periodic solutions of model (31) for case $I_{N} \leq\left(1-q_{1}\right) H_{h}$, where $H_{h}=0.45, H_{l}=0.25$, $q_{1}=q_{2}=0.3, I_{N}=0.2985<\left(1-q_{1}\right) H_{h}=0.3150$, and $p=0.8$, respectively.

trajectory $\pi_{N}(t)$ starting from the initial point $N(0,0.2985)$ which is tangent to line $I=H_{l}$ at point $O_{2}=\left(S_{2}^{*}, H_{l}\right)$, as shown in Figure 3(a). Obviously, $I_{N}=0.2985<\left(1-q_{1}\right) H_{h}=$ 0.3150 . Now, we take $p=0.8$; it is easy to calculate that

$$
\begin{aligned}
|\mu| & =\frac{1-\phi(T)}{1-(1-p) \phi(T)}\left|\frac{(1-p)\left(\beta e^{-\alpha\left(1-q_{1}\right) H_{h}}(1-p) \phi(T)-\mu-\gamma\right)}{\beta e^{-\alpha H_{h}} \phi(T)-\mu-\gamma}\right| \\
& =\frac{1-0.2732}{1-(1-0.8) \times 0.2732}\left|\frac{(1-0.8)\left(0.8 e^{-0.5 \times(1-0.3) \times 0.45}(1-0.8) \times 0.2732-0.1-0.04\right)}{0.8 e^{-0.5 \times 0.45} \times 0.2732-0.1-0.04}\right| \approx 0.4572<1 .
\end{aligned}
$$

Therefore, model (31) has two order-1 periodic solutions which are orbitally asymptotically stable by Theorem 11 and
Corollary 12. Numerical simulations in Figures 3(a)-3(d) show that model (31) has two positive order-1 periodic 


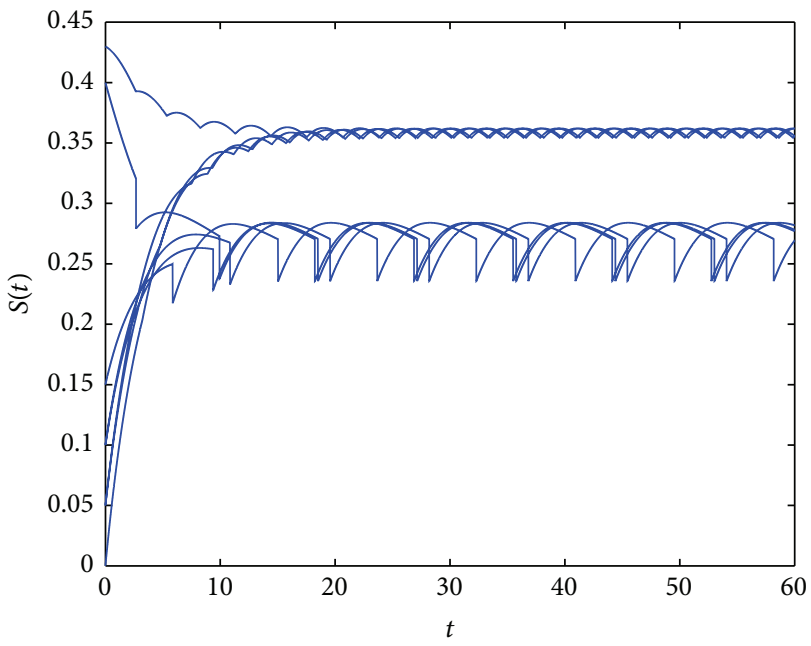

(a)

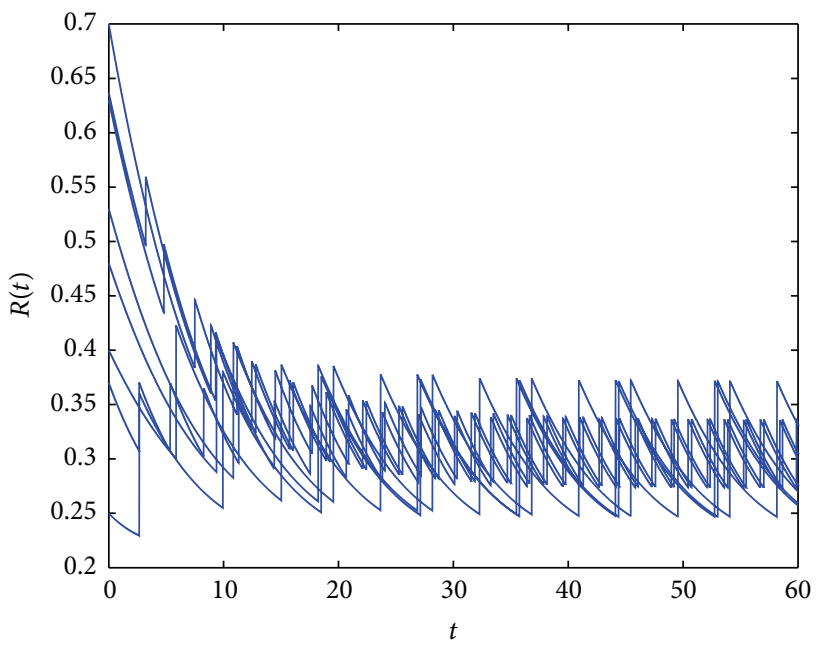

(c)

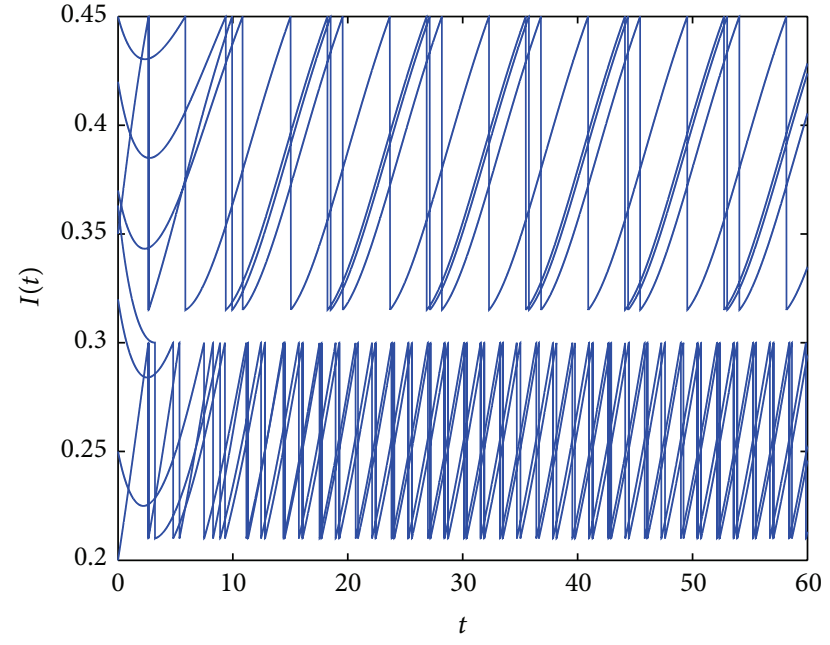

(b)

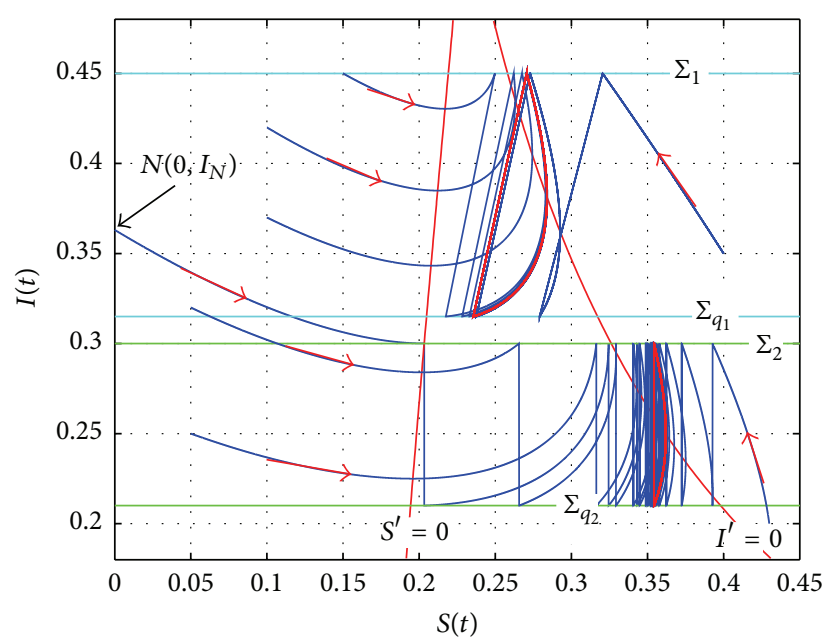

(d)

FIGURE 4: The existence and orbital stability of order- 1 periodic solutions of model (31) for case $I_{N}>\left(1-q_{1}\right) H_{h}$, where $H_{h}=0.45, H_{l}=0.3$, $q_{1}=q_{2}=0.3, I_{N}=0.3631>\left(1-q_{1}\right) H_{h}=0.3150$, and $p=0.1294$.

solutions: one starts from point $(0.3936,0.1750) \in \Sigma_{q_{2}}$, and the other starts from point $(0.0546,0.3150) \in \Sigma_{q_{1}}$, which are orbitally asymptotically stable.

Nextly, we choose control parameter $H_{l}=0.3<(1-$ $\left.q_{1}\right) H_{h}=0.315$ and other parameters fixed as above. It is easy to calculate that $S_{1}^{*}=(\mu+\gamma) e^{\alpha H_{h}} / \beta=0.2192$ and $S_{2}^{*}=(\mu+\gamma) \exp \left(\alpha H_{l}\right) / \beta=0.2033$. By numerical simulations, model (31) has a trajectory which starts from the initial point $(0,0.3631)$ and is tangent to line $I=H_{l}$. This is what show in Figure 4(d). Obviously, $I_{N}=0.3631>\left(1-q_{1}\right) H_{h}=0.3150$. It can be easily calculated that

$$
\begin{gathered}
\theta_{1}^{*}=S_{2}^{*}-\left(\mu-\beta e^{-\alpha\left(1-q_{1}\right) H_{h}} S_{2}^{*}\left(1-q_{1}\right) H_{h}-\mu S_{2}^{*}\right) \\
\cdot \frac{1}{\mu+\gamma} \ln \frac{\left(1-q_{1}\right) H_{h}}{H_{l}}=0.2033-(0.1
\end{gathered}
$$

$$
\begin{aligned}
& \left.-0.8 e^{-0.5 \times 0.21} \times 0.2033 \times 0.21-0.1 \times 0.2033\right) \frac{1}{0.14} \\
& \cdot \ln \frac{0.7 \times 0.45}{0.3}=0.1908 \\
& 1-\frac{\beta}{\mu+\gamma} \theta_{1}^{*} e^{-\alpha H_{h}}=1-\frac{0.8}{0.14} \times 0.1908 \times e^{-0.5 \times 0.45} \\
& =0.1294 .
\end{aligned}
$$




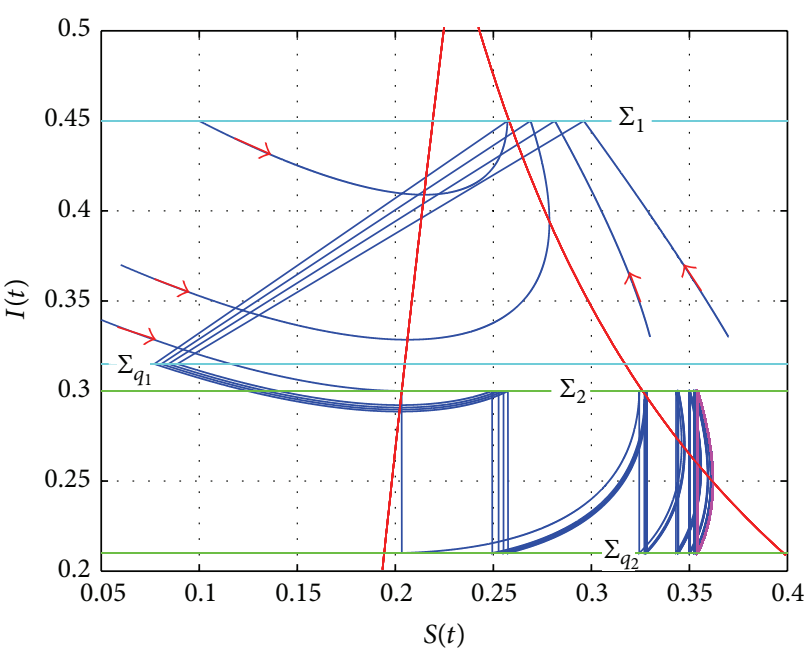

(a)

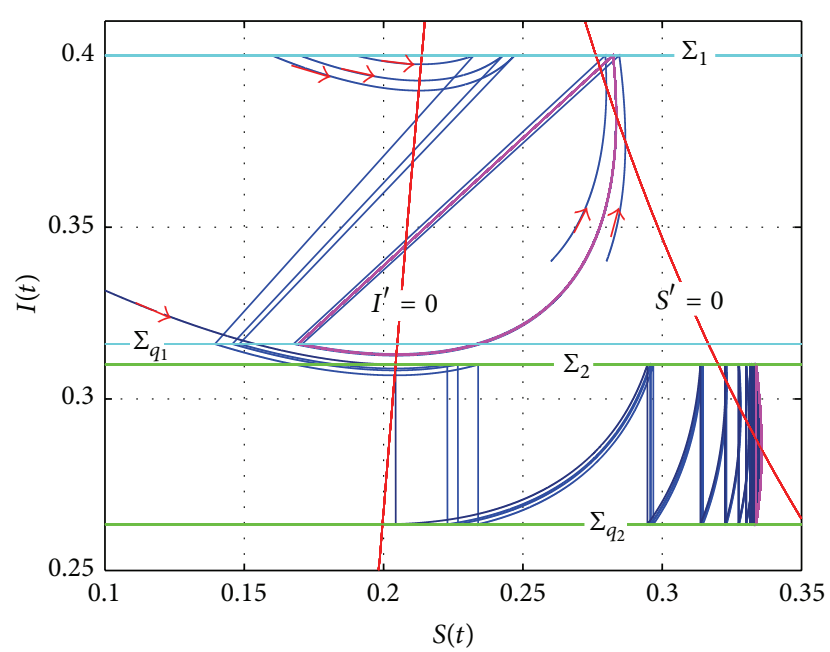

(b)

FIgURE 5: The complex dynamical behaviors of model (31) due to the effects of state dependent pulse control strategies, where (a) $H_{h}=0.45$, $H_{l}=0.3, q_{1}=q_{2}=0.3$, and $p=0.8$; (b) $H_{h}=0.4, H_{l}=0.31, q_{1}=0.21, q_{2}=0.15$, and $p=0.4$.

$$
\begin{aligned}
|\mu| & =\frac{1-\phi(T)}{1-(1-p) \phi(T)}\left|\frac{(1-p)\left(\beta e^{-\alpha\left(1-q_{1}\right) H_{h}}(1-p) \phi(T)-\mu-\gamma\right)}{\beta e^{-\alpha H_{h}} \phi(T)-\mu-\gamma}\right| \\
& =\frac{1-0.2706}{1-0.8706 \times 0.2706}\left|\frac{0.8706 \times\left[0.8 e^{-0.5 \times(1-0.3) \times 0.45} \times 0.8706 \times 0.2706-0.1-0.04\right]}{0.8 e^{-0.5 \times 0.45} \times 0.2706-0.1-0.04}\right| \approx 0.5309<1 .
\end{aligned}
$$

Therefore, model (31) has two positive order-1 periodic solutions which are orbitally asymptotically stable by Theorem 13 . The plots in Figures 4(a)-4(d) show that one orbital stable order-1 periodic solution starts from point $(0.3543,0.21) \epsilon$ $\Sigma_{q_{2}}$, and the other starts from point $(0.2356,0.315) \in \Sigma_{q_{1}}$.

We choose, finally, the vaccination intensity $p=0.8>1-$ $\beta \theta_{1}^{*} e^{-\alpha H_{h}} /(\mu+\gamma)=0.1294$. Then, the condition of Theorem 13 cannot be met. The plots in Figure 5(a) show that model (31) only has a positive order-1 periodic solution starting from set $\Sigma_{q_{2}}$. We choose, however, control parameters to be $H_{h}=0.4$, $H_{l}=0.31, q_{1}=0.21$, and $q_{2}=0.15$. From numerical simulation and calculation, we have that $I_{N}=0.37635>$ $\left(1-q_{1}\right) H_{h}=0.3160$ and $\theta_{2}^{*}=0.1994$. By Theorem 13, we can know that model (31) has two positive order-1 periodic solutions satisfying $0<p \leq 1-\beta \theta_{2}^{*} e^{-\alpha H_{h}} /(\mu+\gamma)=$ 0.0671 . In particular, take $p=0.4>0.0671$ which does not satisfy the condition of Theorem 13, but Figure 5(b) shows that model (31) has two positive order-1 periodic solutions. This implies that the dynamical behaviors of model (31) are complex because of the effects of state dependent pulse control strategies.

\section{Concluding Remarks}

Seeking a reasonable and valid control strategy to prevent infectious diseases from spreading, a novel SIR epidemic model with media impact and state dependent pulse control strategies is proposed. This model is totally different from the traditional state dependent pulse differential equation, where we consider the influences of media impact on people behaviors. That is, to different threat levels and different stages of disease, people's mind and behaviors are different. Therefore, we introduce novel control strategies which are dependent not only on the state of disease, but also on the mind and behaviors of people.

By using the methods of qualitative and successor function, we have studied the existence and orbital stability of positive order-1 periodic solutions of model (7) for various cases. The theoretical results show that model (7) always has order-1 periodic solution starting from pulse set $\Sigma_{q_{2}}$, which is orbitally asymptotically stable for the initial value $\left(S_{0}, I_{0}\right) \in$ $\Omega_{2} \cup \Omega_{3}$ (see Theorem 10), and exists two positive order-1 periodic solutions in region $\Omega_{1} \cup \Omega_{2}$ with condition $I_{N} \leq$ $\left(1-q_{1}\right) H_{h}$ or $I_{N}>\left(1-q_{1}\right) H_{h}$. This needs some condition guarantee (see Theorem 11 or Theorem 13).

Theoretical results and numerical simulations, in this paper, show that state dependent pulse control strategies are feasible and effective to prevent and control the spread of infectious disease. We can control the density of infected individuals at a low level over a long period of time by adjusting immune, medication strength or monitoring threshold values. At the same time, numerical simulations also show 
that model (7) has richer dynamical behaviors because of the effects of state dependent pulse control strategies.

\section{Conflict of Interests}

The authors declare that there is no conflict of interests regarding the publication of this paper.

\section{Acknowledgments}

This research has been partly supported by the National Natural Science Foundation of China (Grants nos. 11461067, 11402223, and 11271312) and the China Postdoctoral Science Foundation (Grants nos. 2012T50836 and 20110491750).

\section{References}

[1] R. Anderson and R. May, Infectious Disease of Humans, Dynamics and Control, Oxford University Press, New York, NY, USA, 1995.

[2] S. J. Gao, L. S. Chen, and Z. D. Teng, "Impulsive vaccination of an SEIRS model with time delay and varying total population size," Bulletin of Mathematical Biology, vol. 69, no. 2, pp. 731745, 2007.

[3] S. J. Gao, L. S. Chen, J. J. Nieto, and A. Torres, "Analysis of a delayed epidemic model with pulse vaccination and saturation incidence," Vaccine, vol. 24, no. 35-36, pp. 6037-6045, 2006.

[4] A. J. Terry, "Pulse vaccination strategies in a metapopulation SIR model," Mathematical Biosciences and Engineering, vol. 7, no. 2, pp. 455-477, 2010.

[5] A. d'Onofrio, "Stability properties of pulse vaccination strategy in SEIR epidemic model," Mathematical Biosciences, vol. 179, no. 1, pp. 57-72, 2002.

[6] G. R. Jiang and Q. G. Yang, "Periodic solutions and bifurcation in an SIS epidemic model with birth pulses," Mathematical and Computer Modelling, vol. 50, no. 3-4, pp. 498-508, 2009.

[7] S. Alonso-Quesada, M. de la Sen, R. P. Agarwal, and A. Ibeas, "An observer-based vaccination control law for an SEIR epidemic model based on feedback linearization techniques for nonlinear systems," Advances in Difference Equations, vol. 2012, article 161, 2012.

[8] B. Shulgin, L. Stone, and Z. Agur, "Pulse vaccination strategy in the SIR epidemic model," Bulletin of Mathematical Biology, vol. 60, no. 6, pp. 1123-1148, 1998.

[9] G. Röst and Z. Vizi, "Backward bifurcation for pulse vaccination," Nonlinear Analysis: Hybrid Systems, vol. 14, pp. 99-113, 2014.

[10] S. Y. Tang, Y. N. Xiao, and D. Clancy, "New modelling approach concerning integrated disease control and cost-effectivity," Nonlinear Analysis, Theory, Methods and Applications, vol. 63, no. 3, pp. 439-471, 2005.

[11] L. F. Nie, Z. D. Teng, and A. Torres, "Dynamic analysis of an SIR epidemic model with state dependent pulse vaccination," Nonlinear Analysis: Real World Applications, vol. 13, no. 4, pp. 1621-1629, 2012.

[12] L.-F. Nie, Z.-D. Teng, and B.-Z. Guo, "A state dependent pulse control strategy for a SIRS epidemic system," Bulletin of Mathematical Biology, vol. 75, no. 10, pp. 1697-1715, 2013.

[13] G. Jiang and Q. Lu, "The dynamics of a prey-predator model with impulsive state feedback control," Discrete and Continuous Dynamical Systems Series B, vol. 6, no. 6, pp. 1301-1320, 2006.
[14] S. Tang, Y. Xiao, L. Chen, and R. A. Cheke, "Integrated pest management models and their dynamical behaviour," Bulletin of Mathematical Biology, vol. 67, no. 1, pp. 115-135, 2005.

[15] T. T. Zhao, Y. N. Xiao, and R. J. Smith, "Non-smooth plant disease models with economic thresholds," Mathematical Biosciences, vol. 241, no. 1, pp. 34-48, 2013.

[16] G. P. Pang and L. S. Chen, "Periodic solution of the system with impulsive state feedback control," Nonlinear Dynamics, vol. 78, pp. 743-753, 2014.

[17] R. S. Liu, J. H. Wu, and H. P. Zhu, "Media/psychological impact on multiple outbreaks of emerging infectious diseases," Computational and Mathematical Methods in Medicine, vol. 8, no. 3, pp. 153-164, 2007.

[18] J. M. Tchuenche, N. Dube, C. P. Bhunu, R. J. Smith, and C. T. Bauch, "The impact of media coverage on the transmission dynamics of human influenza," BMC Public Health, vol. 11, no. 1, article S5, 2011.

[19] J. A. Cui, Y. H. Sun, and H. P. Zhu, "The impact of media on the control of infectious diseases," Journal of Dynamics and Differential Equations, vol. 20, no. 1, pp. 31-53, 2008.

[20] A. Wang and Y. Xiao, "A Filippov system describing media effects on the spread of infectious diseases," Nonlinear Analysis: Hybrid Systems, vol. 11, no. 1, pp. 84-97, 2014.

[21] C. Sun, W. Yang, J. Arino, and K. Khan, "Effect of mediainduced social distancing on disease transmission in a two patch setting," Mathematical Biosciences, vol. 230, no. 2, pp. 87-95, 2011.

[22] V. Lakshmikantham, D. D. Bainov, and P. S. Simeonov, Theory of Impulsive Differential Equations, World Scientific, Singapore, 1989.

[23] J. Hale and H. Kocak, Dynamics and Bifurcations, Springer, New York, NY, USA, 1991.

[24] P. S. Simeonov and D. D. Bainov, "Orbital stability of periodic solutions of autonomous systems with impulse effect," International Journal of Systems Science, vol. 19, no. 12, pp. 2561-2585, 1988. 


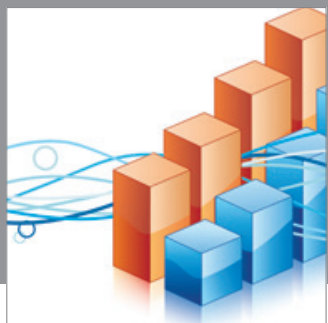

Advances in

Operations Research

mansans

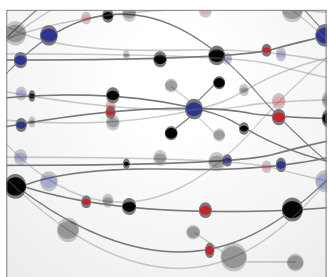

The Scientific World Journal
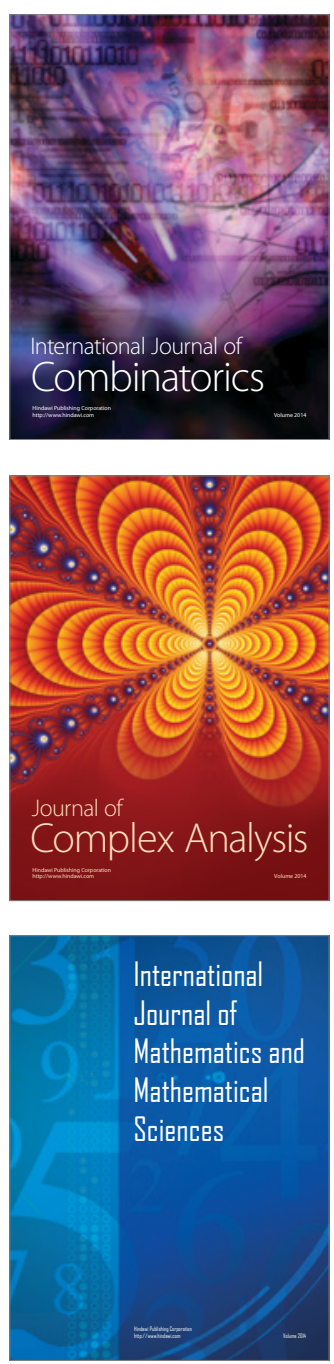
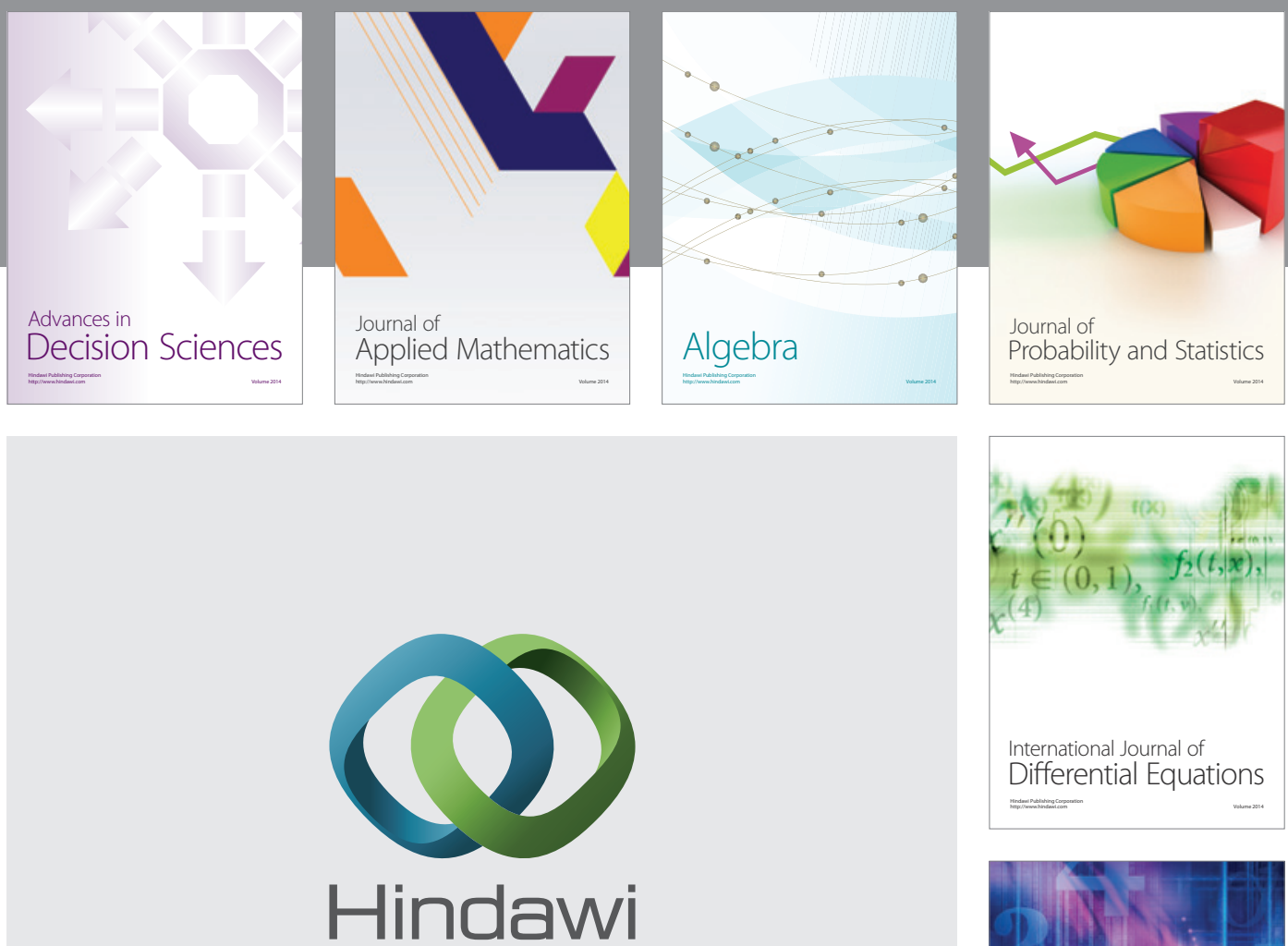

Submit your manuscripts at http://www.hindawi.com
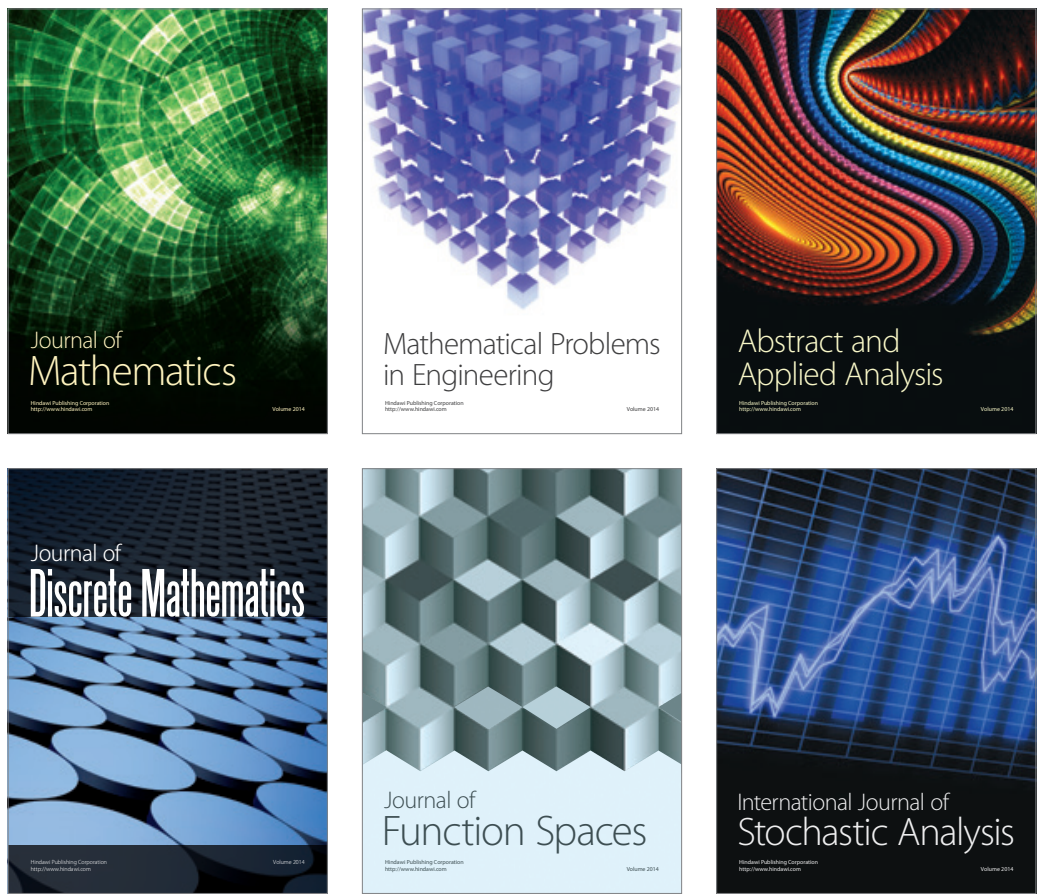

Journal of

Function Spaces

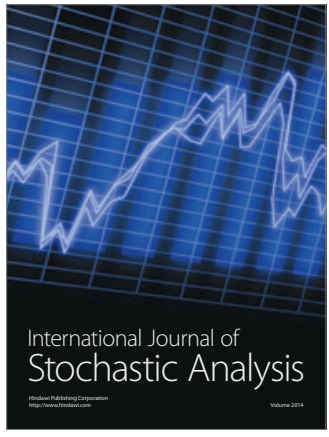

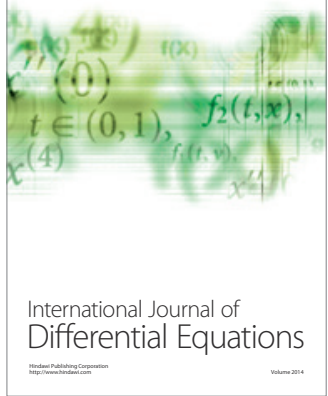
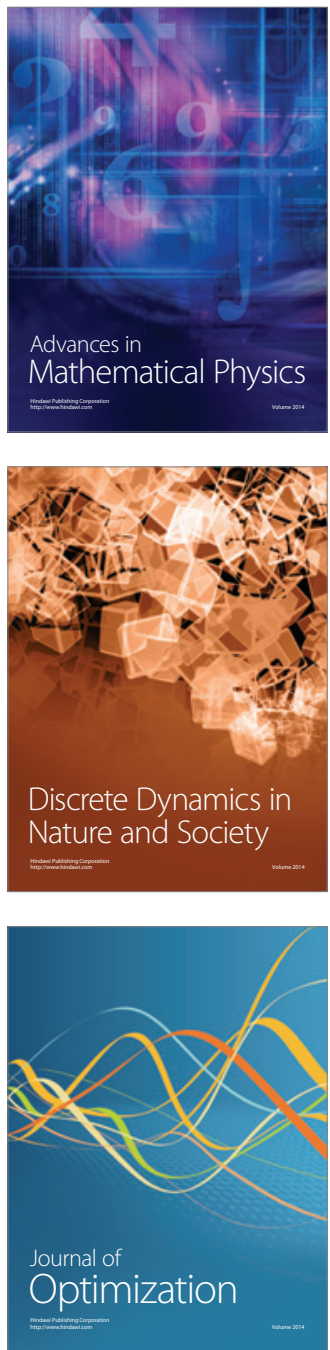